\title{
Alcohol abuse and ERP components in Go/No-go tasks using alcohol-related stimuli: Impact of alcohol avoidance
}

\author{
Fanny Kreusch, Etienne Quertemont, Aurélie Vilenne, Michel Hansenne* \\ Department of Psychology, University of Liège, Belgium
}

\section{A R T I C L E I N F O}

\section{Article history:}

Received 29 May 2014

Received in revised form 11 July 2014

Accepted 3 August 2014

Available online 9 August 2014

\section{Keywords:}

Alcohol

Inhibition

Alcohol-related cues

Go/No-go

ERPS

\begin{abstract}
A B S T R A C T
Alcohol addictive behaviors are associated with a combination of deficits in executive functions, such as a weak response inhibition, and potent automatic appetitive responses to alcohol-related cues. The aim of the present study was to investigate behavioral responses and event-related potentials (ERPs) associated with specific response inhibition for alcohol-related cues. Thirty participants (15 heavy drinkers and 15 light drinkers) took part in the study. Response inhibition was assessed by a classical letter Go/No-go task and by a modified alcohol Go/No-go task. Participants were also classified as high and low alcohol avoiders. Results showed that heavy drinkers made more false alarms in the letter Go/No-go task. In the alcohol Go/No-go task, an absence of N200 amplitude anteriorization was found in heavy drinkers as compared to light drinkers. Participants with a high level of alcohol avoidance exhibited more false alarms, and higher N200 amplitude for the No-go trials as compared to the Go trials for alcohol-related cues. Higher P300 amplitude was observed in low alcohol avoiders for No-go as compared to Go trials. Therefore, a context involving alcohol-related cues disturbed inhibition capacities of high alcohol avoiders. These results suggest that the level of alcohol avoidance must be taken into account in studies investigating alcohol-related cognitive biases.
\end{abstract}

(c) 2014 Elsevier B.V. All rights reserved.

\section{Introduction}

Alcohol consumption leads to several cognitive deficits, either temporary, due to the acute intoxication or permanent, following a chronic use. According to the dual process theory (Wiers et al., 2007; Stacy and Wiers, 2010), chronic alcohol use induces a sensitization of the appetitive system in combination with an increase of the salience of alcoholrelated cues (Robinson and Berridge, 1993) as well as a progressive decline of the regulatory executive system (Parsons, 1998), as evidenced particularly by a lack of executive resources required to inhibit inappropriately salient responses to alcohol. Indeed, several studies reported that alcohol abusers show an attentional bias (Field et al., 2007; Townshend and Duka, 2001) as well as automatic approach behaviors towards alcohol-related cues (Field et al., 2008; Wiers et al., 2007). These two processes (i.e., altered inhibition capacities and increased attentional bias for alcohol) seem to be involved in the development of alcohol-seeking behaviors (Fadardi and Cox, 2008; Nigg et al., 2006) and in alcohol relapse (Field and Cox, 2008).

Inhibition capacities are often studied with a Go/No-go task. In this task, participants are required to respond by pressing a key when

\footnotetext{
* Corresponding author at: Department of Psychology: Cognition and Behavior, University of Liège, 5, Boulevard du Rectorat (B32), B-4000 Liège, Belgium. Tel.: + 324 36623 90; fax: + 3243662859 .

E-mail address: michel.hansenne@ulg.ac.be (M. Hansenne).
}

frequent Go stimuli are presented and to withhold their responses for infrequent No-go stimuli. This paradigm typically gives rise to larger N200 and P300 amplitudes during No-go trials as compared to Go trials (Bruin and Wijers, 2002; Smith et al., 2008). While the frontal N200 would reflect a general control process during No-go trials (Nieuwenhuis et al., 2003), there are debates over its possible role in the inhibition of a premature response (e.g., Falkenstein et al., 1999; Lavric et al., 2004), in the conflict monitoring between execution and inhibition of a single response (Nieuwenhuis et al., 2003; Yeung and Cohen, 2006) or in neither of these (Smith et al., 2007). The frontocentral P300 would index the late decision process to inhibit the motor response (Smith et al., 2010; Gajewski \& Falkenstein, 2013; Huster et al., 2013).

In alcohol-dependent patients, reduced N200 peak amplitude for Go as well as No-go trials has been observed in comparison to controls in an equiprobable Go/No-go task (Pandey et al., 2012). Moreover, in line with the lack of inhibition observed following chronic alcohol consumption using behavioral tasks (Fillmore, 2003; Noël et al., 2010), several studies have found smaller P300 amplitude during No-go trials in alcohol-dependent patients (Cohen et al., 1997; Kamarajan et al., 2005). Similar to alcohol-dependent patients, heavy social drinkers exhibit a reduction of the No-go P300 amplitude compared to light drinkers (Oddy and Barry, 2009). Lack of inhibitory skills have also been associated with compensatory neuronal mechanisms allowing drinkers to achieve performance levels similar to those in controls with increased No-go P3 amplitude and higher prefrontal activation in 
binge drinkers, as compared to controls, when successful inhibition responses were analyzed (López-Caneda et al., 2012).

Interestingly, many studies have reported specific ERP patterns in chronic alcohol users confronted to alcohol-related cues. Most studies conducted on individuals with low sensitivity to alcohol, on heavy drinkers (i.e., more than 4 drinks on any day or 14 per week and usually classified as score higher than 11 at the Alcohol Use Disorders Identification Test, AUDIT; Fleming et al., 1991) and on alcohol-dependent patients have shown larger P300 amplitudes and shorter P300 latencies elicited by alcohol-related pictures or alcohol-related words as compared to neutral stimuli (Bartholow et al., 2007, 2010; Hansenne et al., 2003; Herrmann et al., 2000, 2001; Namkoong et al., 2004). These results suggest that alcohol-related cues are more salient for chronic alcohol users. In contrast, college binge drinkers showed similar P300 amplitudes elicited by alcohol-related pictures during the first stage of development of an abusive use of alcohol as compared to non-binge drinkers (Petit et al., 2012b). However, greater P100 amplitudes to alcoholic stimuli as compared to neutral stimuli in young social drinkers were found in this study, suggesting enhanced perceptual processing toward alcohol cues.

Taken together, these results indicate that poor inhibition responses and automatic processes of alcohol-related cues in heavy drinkers could be reflected by modulations of the N200 and P300 amplitudes. However, only a few studies have investigated the effects of inhibition deficits towards alcohol-related cues, most researches to date mainly focused on the appetitive or attractive value of alcohol-related cues. Nevertheless, from a clinical point of view, being able to inhibit the consumption of an alcoholic beverage when exposed to alcohol-related cues is very important for alcohol-dependent patients to maintain abstinence, and also for heavy drinkers who wish to regulate their consumption. Indeed, it could be assumed, similarly to alcohol-dependent patients, that heavy drinkers are less able to inhibit a response toward alcohol-related cues that capture attention automatically. However, two studies did not observe such a deficit in heavy social drinkers (Adams et al., 2013; Nederkoorn et al., 2009), whereas detoxified alcohol-dependent patients showed impaired response inhibition towards alcohol-related cues after 19.7 ( \pm 2.7 ) days of abstinence (Noël et al., 2007). However, several ERP studies revealed early cerebral dysfunctions related to alcohol consumption before any detectable behavioral impairment in social drinkers (Bijl et al., 2005; Nichols and Martin, 1996; Oddy and Barry, 2009), and in binge drinkers (López-Caneda et al., 2013, 2014; Maurage et al., 2009, 2012; Watson et al., 2014). To our knowledge, only one electrophysiological study assessed the inhibition abilities toward alcohol-related information (Petit et al., 2012a). The authors used a letter Go/No-go task in which the letters were superimposed on a background picture displaying three different emotional contexts (neutral, alcohol-related, and non-alcohol-related) among heavy and light drinkers. Results showed higher false alarm (FA) errors (i.e., erroneously pressing the key) and a delayed P300 latency in heavy drinkers during No-go trials associated with an alcohol-related context as compared to a non-alcohol context. This suggests less attentional allocation to the Go/No-go task in favor of alcohol-related background information.

The aim of the present study was to assess inhibition capacity and alcohol-cue reactivity among heavy and light drinkers with a classic letter Go/No-go task. In addition, the participants performed a modified Go/No-go task in which they had to inhibit a response towards neutral or alcohol-related pictures. We first hypothesized that heavy drinkers, by comparison to light drinkers, would exhibit inhibition deficits revealed by more FA during No-go trials involving letters as well as reduced N200 and P300 amplitudes (Kamarajan et al., 2005; Montgomery et al., 2013; Pandey et al., 2012). Second, in the modified Go/No-go task, we expected reduced inhibition performances towards alcohol-related cues (Noël et al., 2007; Petit et al., 2012a) combined with delayed P300 latencies (Petit et al., 2012a) in heavy drinkers as compared to light drinkers. Third, we assumed that heavy drinkers would display larger P300 amplitudes than light drinkers to alcohol-related pictures, reflecting higher emotional salience of these stimuli for alcohol abusers (Herrmann et al., 2001). As the present study assesses response inhibition towards alcohol stimuli, approach and avoidance to alcohol, subjective craving and impulsivity were assessed.

\section{Materials and methods}

\subsection{Participants}

Participants were recruited at the Faculty of Psychology of the University of Liège (Belgium) through interviews and online advertisements. They were prescreened with the Alcohol Use Disorder Identification Test (AUDIT) and either discarded or assigned into one of two groups according to their reported consumption of alcoholic beverages: light drinkers (AUDIT score $\leq 6$; see Saunders et al., 1993) or heavy drinkers (AUDIT scores $\geq 11$, see Fleming et al., 1991). Participants whose AUDIT scores were comprised between the two limits were excluded from the study as well as alcohol abstainers. Participants included had no major medical problem, no history of central nervous system disorders (including epilepsy and brain trauma), no regular use of drugs other than alcohol and nicotine, no uncorrected serious vision issue (e.g. color blindness), and had French as native language. The final sample comprised 30 participants aged between 18 and 33 years old. The two groups $(\mathrm{N}=15)$ were matched on age and gender (eight females in each group). The groups' characteristics are shown in Table 1. No differences between groups were found regarding levels of anxiety and depression, which is of importance as some studies emphasized relationships between inhibitory control and negative affect states (Kaiser et al., 2003). The study was approved by the Ethic Committee of the Faculty of Psychology of the University of Liège and all participants gave their signed informed consent before taking part in the experiment.

\subsection{Questionnaires}

\subsubsection{Alcohol consumption}

Alcohol consumption was assessed with a self-reported measure based on the timeline follow-back method (Sobell and Sobell, 1990). Participants reported how many standard alcohol drinks (i.e., $10 \mathrm{~g}$ ) they had consumed during the previous week (Wiers et al., 1997). Furthermore, the number of days they drank more than six drinks of alcohol in one occasion during the past 2 weeks was recorded (Wiers et al., 1997).

\section{Table 1}

Mean (standard deviations) for each demographic, psychological and alcohol consumption characteristics in heavy and light drinkers, $t$ value of the Student's test comparison between group and the associate probability.

\begin{tabular}{lllll}
\hline & $\begin{array}{l}\text { Light drinkers } \\
(\mathrm{N}=15)\end{array}$ & $\begin{array}{l}\text { Heavy drinkers } \\
(\mathrm{N}=15)\end{array}$ & $\mathrm{t}$ & $\mathrm{p}$ \\
\hline Age & $21.2(3.9)$ & $21.7(1.8)$ & 0.5 & 0.64 \\
Educational level & $14.1(2.9)$ & $14.8(1.8)$ & 0.8 & 0.41 \\
AUDIT score & $4.0(2.1)$ & $19.5(6.1)$ & 9.3 & $<0.0001$ \\
$\geq 6$ units in one & $0.1(0.3)$ & $1.5(1.3)$ & 3.9 & $<0.001$ \\
$\quad$ occasion/last 2 weeks & & & & \\
Mean number of & $2.3(2.6)$ & $18.5(15.1)$ & 4.1 & $<0.001$ \\
$\quad$ consumption per week & & & & \\
STAI-A & $32.7(8.3)$ & $35.1(4.8)$ & 0.9 & 0.34 \\
STAI-B & $45.5(9.5)$ & $40.7(8.9)$ & -1.4 & 0.16 \\
BDI & $9.9(9.4)$ & $8.3(6.4)$ & -0.5 & 0.61 \\
AAAQ compulsive & $0.5(0.6)$ & $1.5(1.3)$ & 2.8 & 0.02 \\
AAAQ mean urge & $1.6(1.3)$ & $2.5(1.7)$ & 1.1 & 0.3 \\
AAAQ avoidance & $2.2(1.3)$ & $2.6(1.5)$ & 0.9 & 0.4 \\
BIS-II cognitive & $16.1(2.7)$ & $17.8(3.5)$ & 1.3 & 0.19 \\
BIS-II motor & $17.7(3.1)$ & $21.3(3.4)$ & 2.9 & 0.006 \\
BIS-II non planning & $21.3(4.2)$ & $24.2(3.7)$ & 2.0 & 0.05 \\
\hline
\end{tabular}




\subsubsection{Alcohol use disorder identification test}

The AUDIT (Saunders et al., 1993) includes 10 multiple-choice items measuring alcohol consumption, drinking behaviors and alcoholrelated problems. This measure was used to assign the participants into the two groups (light and heavy drinkers, see above).

\subsubsection{Impulsiveness}

The Barrat Impulsiveness Scale-11 (BIS-11) is a 30-item self-report questionnaire measuring the behavioral construct of impulsiveness. It consists of three subscales: motor impulsiveness, cognitive impulsiveness and non-planning impulsiveness. Each item consisted of a 4-point Likert scale from "rarely/never" (1) to "almost always" (4).

\subsubsection{Approach and avoidance}

The Approach and Avoidance of Alcohol Questionnaire (AAAQ McEvoy et al., 2004) is a 14-item self-report questionnaire developed to assess the compulsive urge to drink alcohol and the avoidance towards alcohol. Participants had to indicate the extent to which they agreed or not with each item on a 9-point Likert scale ranging from "not at all" (0) to "very strongly" (8).

\subsubsection{Depression}

The Beck Depression Inventory (BDI-II, Beck et al., 1996) is a 21-item self-report questionnaire used to screen for depressive symptoms experienced during the past 2 weeks. Extensive reliability and validity data have been reported (Beck et al., 1996).

\subsubsection{Anxiety}

The State-Trait Anxiety Inventory for adults form Y (STAI form Y, Spielberger, 1983) is a 40-item self-report questionnaire used to assess the state anxiety (how people feel now) (20 items) and the trait anxiety (how people usually feel, independently of the situation) (20 items).

\subsection{Procedure}

Participants were tested in a sound-attenuated room. They filled in the four questionnaires (AAAQ, BDI, BIS, STAI) before taking part in two different Go/No-go tasks.

\subsubsection{Letter Go/No-go task}

This task consisted of one training session without electroencephalographic (EEG) recording (20 trials, 15 Go trials and 5 No-go trials) and one test session ( 100 trials, 75 Go trials and 25 No-go trials) during which ERPs were recorded. On each trial, a fixation cross was presented on the center of the screen for $1200 \mathrm{~ms}$ followed by a letter for $500 \mathrm{~ms}$. The Go frequent stimulus was the capital letter "O" (size of $500 \times 400 \mathrm{~mm}$ ) and the No-go infrequent stimulus was the letter "E." The letters were displayed on a 17 -in. monitor, and the participants were seated $1 \mathrm{~m}$ from the screen. They were instructed to press the spacebar, with their dominant hand, as quickly and accurately as possible when they detected the Go target and to withhold their response on the No-go trials.

\subsubsection{Alcohol modified Go/No-go task}

The procedure was exactly the same as in the letter Go/No-go task except that the stimuli were 25 colored pictures of alcohol drinks and 25 colored pictures of neutral objects ( 100 trials, 75 Go trials and 25 No-go trials). The alcohol pictures included bottles of beer and wine, glasses of beer, wine, liquor and cocktails, and the neutral object pictures included objects typically used in the office, such as pen, ruler, stapler, computer mouse, and USB stick (Kreusch et al., 2013). Each individual picture was presented three times during Go trials. We used two versions of this task: one in which the alcohol-related pictures were assigned to Go trial and neutral pictures to No-go trial, and one version in which the stimuli had the reverse status. The order of the two versions was counterbalanced across participants. The pictures were displayed fully on a 17-in. monitor, and the participants were placed $1 \mathrm{~m}$ before the screen.

\subsection{EEG recording}

ERP recording, stimulus presentation and waveform analyses were performed with an ANT system (eeprobe, eevoke and eemagine EEG, respectively). EEG activity was recorded at nine sites according to the 10-20 System (Fp1, Fp2, F3, Fz, F4, C3, Cz, C4 and Pz), using Ag/AgCl electrodes, earlobes for reference and forehead for ground. All sites were cleaned with acetone and abraded to maintain impedances below $10 \mathrm{k} \Omega$. Electrooculogram (EOG) activity was recorded from above the left eye. If $50 \%$ or more of the epochs of a participant contained artifacts, this participant was excluded from ERP analyses. As a result, four participants were rejected from ERP analyses, two heavy drinkers and two light drinkers. Then, approximately $15 \%$ of trials were contaminated by eye movements or muscular artifacts and were discarded. A two-way ANOVA with Stimulus type (alcohol-related vs. neutral) as within-subject variable and Group (light vs. heavy drinkers) as between-subject variable showed that the number of rejected trials was similar in each group and condition (Stimulus type: $\mathrm{F}(1,50)=$ 2.4, $\mathrm{p}=0.12$; Group: $\mathrm{F}(1,50)=2.3 ; \mathrm{p}=0.13$, Stimulus type $\times$ Group: $F(1,50)=0.2, p=0.65)$. The EEG was amplified by batteryoperated amplifiers with a gain of 50,000 and a band pass of 0.16-30 $\mathrm{Hz}$ (Advanced Neuro Technology-ANT Ltd., Enschende, the Nederlands). The EEG was digitized at 256 samples for $900 \mathrm{~ms}$ with a $100 \mathrm{~ms}$ prestimulus baseline. Trials on which the EEG or EOG exceeded $50 \mu \mathrm{V}$ were rejected automatically by the system. N200 and P300 components were defined as the maximum negative or positive peaks within the latency windows of 200-300 and 270-650 ms, respectively, from the onset of each stimulus. The detection of the peaks was performed by individual visual inspection with no automatic detection procedure. After considering artifacts and FA from the initial 25 No-go trials, approximately 21 trials were included on average for each subject (mean: 21.74, SD: 1.05).

\subsection{Statistical analyses}

We carried out separate analyses for the letter Go/No-go and the alcohol modified Go/No-go tasks. For the letter Go/No-go task, the percentage of FA on No-go trials and the average reaction times (RT) on Go trials of the two groups (heavy and light drinkers) were analyzed with Student's t-test. N200 and P300 amplitudes and latencies were analyzed with four separate three-way mixed ANOVAs including Group (heavy vs. light drinkers), Trial type (Go and No-go) and Electrodes (F3-FzF4-C3-Cz-C4-Pz; Oddy and Barry, 2009; Petit et al., 2012a) as factors, with Trial type and Electrode as within-subject variables.

To examine whether the level of alcohol avoidance had an influence on behavioral performance and ERP components within the modify alcohol Go/No-go task, we additionally divided participants $(\mathrm{n}=26)$ into two groups, split at the median (2.2) of the alcohol avoidance mean score: high alcohol avoiders $(\mathrm{n}=13,7$ heavy drinkers and 6 light drinkers) and low alcohol avoiders ( $\mathrm{n}=13,6$ heavy drinkers and 7 light drinkers). The average percentage of FA on No-go trials and the average RT on Go trials were analyzed with three-way mixed ANOVAs including Stimulus type (alcohol-related vs. neutral picture) as within subject factor and Group (heavy vs. light drinker) and Alcohol avoidance (high alcohol avoiders and low alcohol) as between subject factors. Five-way mixed ANOVAs with Group (heavy vs. light drinkers), Alcohol avoidance (high alcohol vs. low alcohol avoiders), Stimulus type (alcohol-related vs. neutral picture), Trial type (Go and No-go), and Electrode (F3-Fz-F4-C3-Cz-C4-Pz; Oddy and Barry, 2009; Petit et al., 2012a) were conducted separately with N200 and P300 amplitudes and latencies as dependent variables.

As appropriate, Tukey's post hoc tests and Greenhouse-Geisser corrections were applied. As the electrode effect was not the main 
focus of this study, only Group $\times$ Electrode interactions are reported. Pearson's correlations were used to test the hypothesis that N200 and P300 amplitudes and latencies (mean amplitudes averaged across seven recorded fronto-central scalp sites: F3, Fz, F4, C3, Cz, C4 and Pz) (Euser and Franken, 2012) in No-go trials for alcohol-related cues (Petit et al., 2012a) are associated with the percentage of FAs to alcohol-related cues, AUDIT scores, BIS-11 scores and the obsessed/ compelled craving for alcohol. All statistical analyses were conducted with Statistica (version 10) for Windows.

\section{Results}

\subsection{Behavioral data}

Only RTs of trials with correct responses were analyzed (i.e., $99 \%$ in the classical Go/No-go task and $97 \%$ in the alcohol modified Go/No-go task). The t-tests computed on the RTs and the percentage of FAs in the letter Go/No-go task revealed no significant difference between heavy and light drinkers on RTs $(\mathrm{t}(28)=-1.4, \mathrm{p}=0.17)$, but a significant difference on FAs $(t(28)=2.24, \mathrm{p}=0.03)$. Heavy drinkers made more FA than light drinkers ( mean $=5.6 \%, \mathrm{SD}=5.4$ in heavy drinkers and mean $=2.1 \%, \mathrm{SD}=2.5$ in light drinkers).

For the modified alcohol Go/No-go task, the three-way mixed ANOVA on RTs showed a significant main effect of Stimulus type $(\mathrm{F}(1,26)=23.98, \mathrm{p}<0.001)$. Post-hoc analysis showed significant faster responses for alcohol-related pictures as compared to neutral ones ( $p<0.001$ ) for the Go trials, independently of the groups. The main effects of Group $(F(1,26)=2.79, \mathrm{p}=0.10)$ and Alcohol avoidance $(F(1,26)=0.005, p=0.94)$ were not significant. There was no significant interaction. The three-way mixed ANOVA with percentage of FAs as dependent variable showed a significant main effect of Alcohol avoidance $(F(1,26)=6.25, p=0.02)$. Post-hoc analysis revealed a significantly higher percentage of FAs in high alcohol avoiders compared to low alcohol avoiders $(\mathrm{p}=0.01)$. No significant main effect of Group $(F(1,26)=0.06, p=0.80)$ or Stimulus type was observed $(F(1,26)=0.65, p=0.43)$. However, there was a significant three-way Stimulus type $\times$ Group $\times$ Alcohol avoidance interaction $(\mathrm{F}(1,26)=4.38, \mathrm{p}=0.046)$. Heavy drinkers categorized as higher alcohol avoiders showed a non-significant tendency to perform more FAs to alcohol related-cues as compared to heavy drinkers classified as lower alcohol avoiders $(p=0.09)$. No such tendency was observed for FAs to neutral cues or in light drinkers.

\section{ERP}

\subsection{Letter Go/No-go task}

\subsubsection{N200 component}

The mixed ANOVA conducted with N200 latency as dependent variable revealed a significant main effect of Trial type $(\mathrm{F}(1,24)=$ $16.75, \mathrm{p}<0.001$ ). The N200 latency was longer on No-go trials than on Go trials $(p<0.001)$. No significant main effect of Group $(\mathrm{F}(1,28)=0.14, \mathrm{p}=0.70)$ nor significant interaction $(\mathrm{F}(2,56)=$ $0.008, \mathrm{p}=0.92$ ) were observed.

With N200 amplitude as dependent variable, the mixed ANOVA revealed no significant main effect of Trial type $(F(1,24)=0.97, p=$ $0.33)$, no significant main effect of Group $(F(1,24)=0.08, p=0.77)$, and no significant Trial type $\times$ Group interaction $(\mathrm{F}(1,24)=0.83, \mathrm{p}=$ 0.37). However, a significant Group $\times$ Electrode interaction was observed $(F(6,144)=3.31, p=0.03)$. While no difference of N200 amplitude was observed in heavy drinkers as regards to electrodes, higher non-significant N200 amplitude was found in frontal electrodes $(\mathrm{F} 3, \mathrm{Fz}, \mathrm{F} 4)$ than parietal and central electrodes $(\mathrm{C} 4, \mathrm{Pz})$ in light drinkers $(\mathrm{p}<0.1)$.

\subsubsection{P300 component}

The repeated ANOVA conducted with P300 latency as dependent variable revealed a significant main effect of Trial type $(F(1,24)=$ $17.24, \mathrm{p}<0.001)$. The P300 latency was longer on No-go trials than on Go trials $(\mathrm{p}<0.001)$. No significant Group effect $(\mathrm{F}(1,24)=0.92$, $\mathrm{p}=0.34)$ or interaction $(\mathrm{F}(1,24)=0.002, \mathrm{p}=0.96)$ were observed.

The repeated ANOVA computed with P300 amplitude as dependent variable revealed a significant main effect of Trial type $(F(1,24)=62.79$, $\mathrm{p}<0.001)$ and no significant main effect of Group $(\mathrm{F}(1,24)=0.14, \mathrm{p}=$ $0.71)$ nor Trial type $\times$ Group interaction $(F(1,24)=0.87, \mathrm{p}=0.36)$. The P300 amplitude was higher during No-go trials than during Go trials $(\mathrm{p}<0.001)$.

\section{Alcohol modified Go/No-go task}

\subsection{N200 component}

With N200 latency as dependent variable (see Fig. 1 and Table 2), the mixed ANOVA revealed no significant main effects or interaction.

Regarding N200 amplitude (see Fig. 1 and Table 2), the ANOVA revealed a significant main effect of Trial type $(F(1,22)=10.26, p=$ 0.004). Higher N200 amplitude was observed during No-go trials compared to Go trials ( $\mathrm{p}=0.004)$. There were no other significant main effects. A significant Trial type $\times$ Alcohol avoidance interaction was observed $(\mathrm{F}(1,22)=11.38, \mathrm{p}=0.002)$. We observed a nonsignificant trend showing that drinkers who experienced higher avoidance towards alcohol tended to show a higher N200 during No-go trials compared to drinkers who experienced low alcohol avoidance $(\mathrm{p}=$ 0.057). Moreover, the amplitude of the N200 in drinkers who experienced high avoidance towards alcohol was significantly higher in Nogo trials compared to Go trials ( $\mathrm{p}<0.001$ ) while no such difference was observed in drinkers who experienced less alcohol avoidance $(\mathrm{p}=0.99)$. Finally, a significant Electrode $\times$ Group interaction was observed $(\mathrm{F}(6,132)=5.22, \mathrm{p}=0.006)$. Post-hoc analysis revealed that while no difference of N200 amplitude was observed in heavy drinkers, N200 amplitude was higher at frontal electrodes (F3, Fz, F4) than parietal and central electrodes $(\mathrm{C} 3, \mathrm{C} 4, \mathrm{Pz})$ in light drinkers $(\mathrm{p}<0.01)$. A significant Electrode $\times$ Group $\times$ Trial interaction was also found $(\mathrm{F}(6,132)=3.95, \mathrm{p}=0.02)$.

\subsection{P300 component}

The ANOVA conducted with P300 latency as dependent variable (see Fig. 1 and Table 3 ) revealed a significant main effect of Trial type $(\mathrm{F}(1,22)=48.63, \mathrm{p}<0.001)$ and a significant main effect of Stimulus type $(\mathrm{F}(1,22)=17.32, \mathrm{p}<0.001)$. We found delayed P300 latencies for neutral cues compared to alcohol-related cues and during No-go trials compared to Go trials. No other main effects or interaction were significant.

With P300 amplitude as dependent variable (see Fig. 1 and Table 3), the repeated ANOVA showed a main effect of Trial type $(F(1,22)=$ 23.97, $\mathrm{p}<0.001)$. Post-hoc test indicated higher P300 amplitude during No-go trials as compared to Go trials $(\mathrm{p}<0.001)$. Moreover, we found a significant Stimulus type $\times$ Trial type $\times$ Alcohol avoidance interaction $(\mathrm{F}(1,22)=4.86, \mathrm{p}=0.04)$. Higher $\mathrm{P} 300$ amplitude was observed in low alcohol avoiders for No-go as compared to Go trials for alcoholrelated cues $(p<0.001)$, and no such difference was found in high alcohol avoiders $(p=0.19)$. No other main effects or interaction were significant.

\subsection{Correlations between behavioral data and ERP data}

Correlation analyses showed a positive correlation between the percentages of FA to alcohol-related cues during No-go trials with the impulsivity score $(r=0.43, p=0.02)$ (see Table 4). Analyses also revealed a positive correlation between the percentage of FAs to 
Go Trials
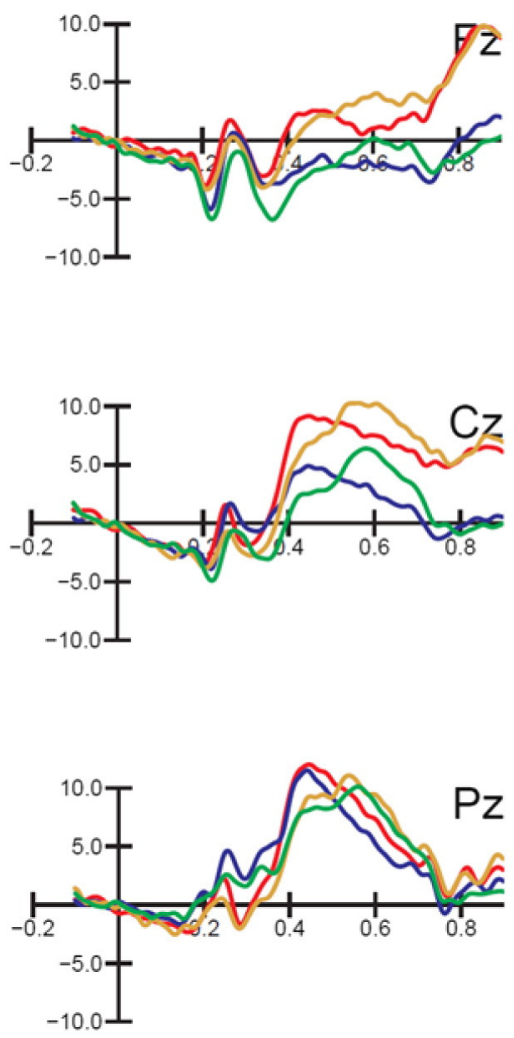

Nogo Trials
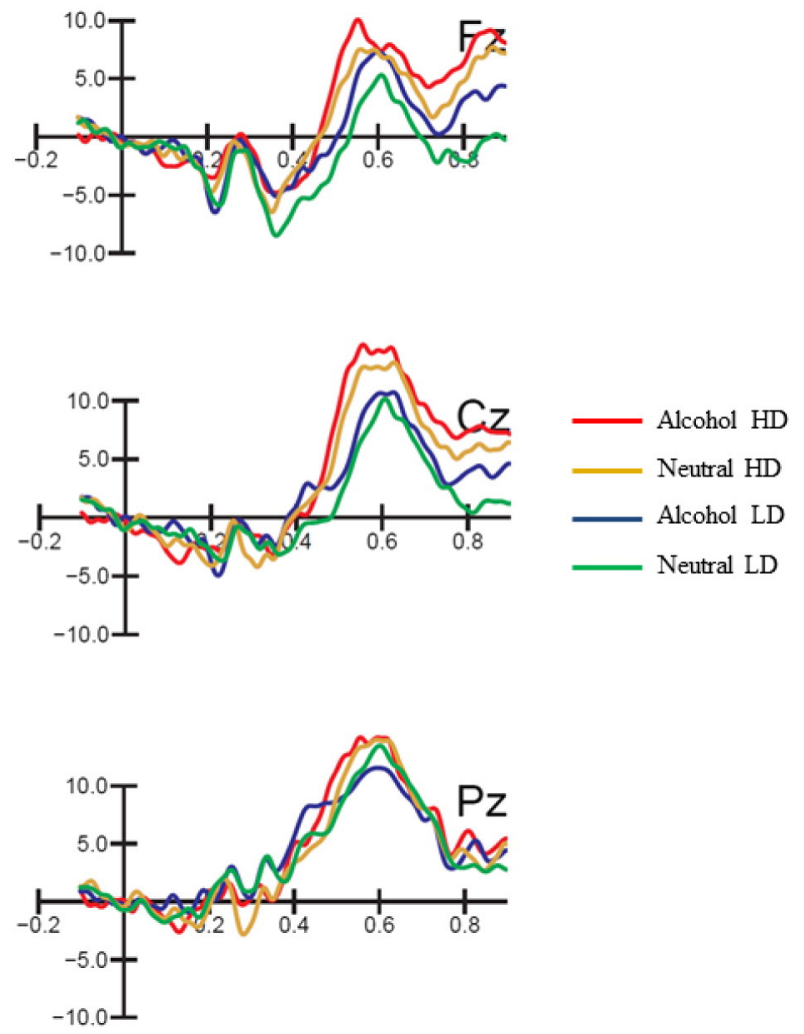

Fig. 1. Illustration of the N200 and P300 on the three central electrodes (FZ, CZ, PZ) in No-go and Go trials. Waves for alcohol-related pictures and neutral pictures in heavy (red and yellow, respectively) and light drinkers (blue and dark green, respectively) are illustrated.

alcohol-related cues and the P300 latency during No-go trials $(r=0.38$, $\mathrm{p}=0.05$ ). However, AUDIT scores, obsessed craving, and impulsivity did not exhibit significant correlations with N200/P300 amplitudes or latencies.

\section{Discussion}

While the improvement of inhibition response has been suggested as a requirement to maintain alcohol consumption under control (Field et al., 2010), the specific exploration of the inhibitory capacities of alcohol-related stimuli in alcohol abuse has received limited attention in the literature. The present study investigated the inhibition processes, and more particularly toward alcohol-related cues, in heavy and light drinkers with a classical letter Go/No-go task and an alcohol modified Go/No-go task, while taking into account the level of alcohol avoidance.

We first hypothesized a general inhibition deficit and reduced N200 and P300 amplitudes in heavy drinkers during the classical letter Go/ No-go task as compared to light drinkers. In line with this hypothesis, heavy drinkers made more FA in this task by comparison with light drinkers. Moreover, based on Group $\times$ Electrode interaction, while no N200 amplitude differences were observed between electrode sites in heavy drinkers, light drinkers showed a non-significant tendency to exhibit higher N200 amplitudes over frontal sites compared to central and parietal electrode. This non-significant tendency was consistent to previous findings (Enriquez-Geppert et al., 2010; Smith et al., 2007). The same pattern of anteriorization among light drinkers was significantly observed in the alcohol modified Go/No-go task. Lower N200 amplitude has previously been observed in detoxified alcohol-dependent patients who performed an equiprobable Go/No-go task as compared to a healthy control group (Pandey et al., 2012). The difference between groups was larger at frontal and central regions, and the N200 was especially affected during No-go trials. In line with these results, our data suggest that the heavy drinkers do not display the N2 anteriorization observed in the light drinkers. Chronic alcoholism may therefore be related to dysfunctional frontal activation associated with control process, particularly when suppression of a motor response is required (i.e., No-go trials).

Contrary to our hypothesis, no difference of P300 amplitude was observed between heavy and light drinkers during No-go trials. Based on the findings of Oddy and Barry (2009), we expected reduced P300 amplitudes during the letter No-go trials in heavy drinkers. Several discrepancies between the two studies could explain the absence of difference in our study. Firstly, in the study of Oddy and Barry (2009), the level of alcohol consumption was based on the number of standard alcoholic drinks per month. Here, heavy and light drinkers were classified according to the AUDIT scores. Since the AUDIT assesses the harmful effects of alcohol consumption, scores might be more influenced by negative consequences of consumption than by the frequency of consumption itself. Secondly, Oddy and Barry (2009) used an equiprobable Go/No-go task in contrast to our task that consisted of $25 \%$ of No-go trials. According to Barry and Rushby (2006), in an equiprobable Go/No-go task, the No-go stimulus is not related to response inhibition process, but rather an involuntary orientation reflex (No-go trials). In contrast, the $75 \% / 25 \%$ distribution used here is more likely to require active inhibition of the response during No-go trials (Barry and Rushby, 2006).

Our second hypothesis concerned an impaired response inhibition towards alcohol-related cues in heavy drinkers. Unlike another study comparing detoxified alcohol-dependent patients to a control group of healthy participants (Noël et al., 2007), we showed no significant difference of inhibition performance or RT towards alcohol-related cues between heavy and light drinkers. This absence of deficit of inhibition 
Table 2

Average N200 amplitudes (in $\mu \mathrm{V}$ ) (standard deviations in parentheses) and latencies (in ms) at F3, Fz, F4, C3, Cz, C4 and Pz as function of group (heavy drinkers with high alcohol avoidance (HD/HAA), heavy drinkers with low alcohol avoidance (HD/LAA), light drinkers with high alcohol avoidance (LD/HAA), light drinkers with low alcohol avoidance (LD/LAA)), type of stimulus (alcohol-related and neutral picture) and type of trial (Go or No-go).

\begin{tabular}{|c|c|c|c|c|c|c|c|c|c|}
\hline & & \multicolumn{4}{|c|}{ Alcohol-related picture } & \multicolumn{4}{|c|}{ Neutral picture } \\
\hline & & \multicolumn{2}{|l|}{ Go trials } & \multicolumn{2}{|c|}{ No-go trials } & \multicolumn{2}{|l|}{ Go trials } & \multicolumn{2}{|c|}{ No-go trials } \\
\hline & & Lat & Amp & Lat & Amp & Lat & Amp & Lat & Amp \\
\hline \multirow[t]{7}{*}{$\mathrm{HD} / \mathrm{HAA}$} & F3 & 296(66) & $-2.9(2.7)$ & $281(81)$ & $-6.1(1.7)$ & $245(55)$ & $-4.4(2.5)$ & $271(55)$ & $-8.8(4.7)$ \\
\hline & $\mathrm{Fz}$ & $299(68)$ & $-4.7(3.7)$ & $280(80)$ & $-8.1(4.5)$ & $268(57)$ & $-5.5(2.5)$ & $268(57)$ & $-9.4(3.6)$ \\
\hline & $\mathrm{F} 4$ & $263(60)$ & $-2.9(2.7)$ & $279(80)$ & $-5.4(1.7)$ & $263(62)$ & $-3.8(1.4)$ & $263(62)$ & $-6.5(3.2)$ \\
\hline & C3 & $272(63)$ & $-4.8(2.9)$ & $254(49)$ & $-6.5(3.7)$ & $272(51)$ & $-4.7(3.3)$ & $272(51)$ & $-8.8(7.4)$ \\
\hline & $\mathrm{Cz}$ & $277(68)$ & $-5.8(2.6)$ & $278(65)$ & $-7.5(3.6)$ & $271(52)$ & $-6.1(2.0)$ & $289(53)$ & $-10.0(5.6)$ \\
\hline & C4 & $265(56)$ & $-3.8(2.4)$ & $291(76)$ & $-5.0(2.5)$ & $286(42)$ & $-3.7(2.3)$ & $284(53)$ & $-6.7(4.4)$ \\
\hline & $\mathrm{Pz}$ & $286(53)$ & $-4.4(3.9)$ & $291(46)$ & $-4.9(3.3)$ & $305(35)$ & $-4.7(4.8)$ & $290(46)$ & $-6.4(6.7)$ \\
\hline \multirow[t]{7}{*}{ HD/LAA } & F3 & $260(54)$ & $-4.4(1.5)$ & $239(54)$ & $-6.8(4.7)$ & $245(36)$ & $-5.3(1.7)$ & $270(67)$ & $-5.8(2.4)$ \\
\hline & $\mathrm{Fz}$ & $258(55)$ & $-5.0(1.2)$ & $239(54)$ & $-8.0(5.3)$ & $252(44)$ & $-5.4(1.6)$ & $272(66)$ & $-7.2(3.4)$ \\
\hline & $\mathrm{F} 4$ & $263(56)$ & $-4.2(2.1)$ & 23958) & $-7.1(5.1)$ & $254(41)$ & $-5.4(2.9)$ & $269(68)$ & $-5.4(2.5)$ \\
\hline & C3 & $245(53)$ & $-4.7(2.1)$ & $243(55)$ & $-5.4(3.1)$ & $245(27)$ & $-6.1(3.3)$ & $268(56)$ & $-5.1(2.3)$ \\
\hline & $\mathrm{Cz}$ & $253(47)$ & $-5.7(2.2)$ & $243(51)$ & $-6.6(4.4)$ & $241(30)$ & $-8.0(5.6)$ & $267(58)$ & $-5.9(2.9)$ \\
\hline & C4 & $246(50)$ & $-4.0(3.4)$ & $234(28)$ & $-4.8(4.3)$ & $251(30)$ & $-6.6(6.4)$ & $265(60)$ & $-3.7(3.9)$ \\
\hline & $\mathrm{Pz}$ & $263(54)$ & $-4.7(3.5)$ & $235(36)$ & $-3.9(5.0)$ & $261(29)$ & $-8.2(7.7)$ & $284(56)$ & $-5.3(4.0)$ \\
\hline \multirow[t]{7}{*}{ LD/HAA } & F3 & $259(43)$ & $-6.4(4.2)$ & $257(48)$ & $-9.6(6.8)$ & $280(61)$ & $-8.9(5.9)$ & $284(53)$ & $-10.1(5.5)$ \\
\hline & $\mathrm{Fz}$ & $243(48)$ & $-7.3(4.2)$ & $256(50)$ & $-10.5(5.6)$ & $279(62)$ & $-10.4(5.5)$ & $283(61)$ & $-11.6(4.9)$ \\
\hline & $\mathrm{F} 4$ & $243(48)$ & $-6.5(3.4)$ & $246(51)$ & $-9.0(5.0)$ & $278(59)$ & $-7.9(4.4)$ & $283(62)$ & $-9.4(4.5)$ \\
\hline & C3 & $230(48)$ & $-3.7(1.9)$ & $250(52)$ & $-5.9(5.5)$ & $269(51)$ & $-5.1(3.2)$ & $261(28)$ & $-6.8(2.9)$ \\
\hline & $\mathrm{Cz}$ & $241(47)$ & $-5.8(3.1)$ & $248(50)$ & $-8.5(5.4)$ & $281(61)$ & $-7.6(3.7)$ & $287(62)$ & $-8.7(3.4)$ \\
\hline & C4 & $225(9)$ & $-4.3(1.7)$ & $249(47)$ & $-6.6(3.2)$ & $264(48)$ & $-5.2(2.5)$ & $265(56)$ & $-5.6(3.8)$ \\
\hline & $\mathrm{Pz}$ & $229(22)$ & $-1.8(2.7)$ & $249(47)$ & $-2.1(4.6)$ & $264(67)$ & $-2.6(2.1)$ & $250(47)$ & $-3.9(2.6)$ \\
\hline \multirow[t]{7}{*}{ LD/LAA } & F3 & $254(74)$ & $-5.7(3.5)$ & $245(48)$ & $-4.9(2.3)$ & $241(48)$ & $-6.5(3.5)$ & $261(65)$ & $-5.8(5.1)$ \\
\hline & $\mathrm{Fz}$ & $255(73)$ & $-7.5(3.1)$ & $252(59)$ & $-7.3(2.7)$ & $241(46)$ & $-7.9(3.9)$ & $261(67)$ & $-7.2(5.0)$ \\
\hline & $\mathrm{F} 4$ & $254(73)$ & $-5.2(2.7)$ & $254(60)$ & $-5.4(2.6)$ & $242(46)$ & $-5.9(2.9)$ & $262(68)$ & $-4.1(3.2)$ \\
\hline & C3 & $262(74)$ & $-2.5(3.7)$ & $259(51)$ & $-2.6(2.4)$ & $257(50)$ & $3.5(2.9)$ & $276(60)$ & $-2.5(4.1)$ \\
\hline & $\mathrm{Cz}$ & $252(74)$ & $-4.1(2.8)$ & $254(57)$ & $-4.9(3.2)$ & $262(59)$ & $5.4(2.6)$ & $272(60)$ & $-3.8(2.7)$ \\
\hline & C4 & $253(70)$ & $-2.0(2.8)$ & $247(48)$ & $-3.0(3.5)$ & $252(50)$ & $-3.1(2.7)$ & $280(58)$ & $-0.8(1.9)$ \\
\hline & $\mathrm{Pz}$ & $279(68)$ & $-0.5(4.2)$ & 293(64) & $-3.0(5.4)$ & $253(57)$ & $-1.5(3.3)$ & $278(53)$ & $-0.9(1.9)$ \\
\hline
\end{tabular}

Table 3

Mean P300 amplitudes (in $\mu \mathrm{V}$ ) (standard deviations in parentheses) and latencies (in ms) at F3, Fz, F4, C3, Cz, C4 and PZ as function of group (heavy drinkers with high alcohol avoidance (HD/HAA), heavy drinkers with low alcohol avoidance (HD/LAA), light drinkers with high alcohol avoidance (LD/HAA), light drinkers with low alcohol avoidance (LD/LAA)), type of stimulus (alcohol-related and neutral picture) and type of trial (Go or No-go).

\begin{tabular}{|c|c|c|c|c|c|c|c|c|c|}
\hline & & \multicolumn{4}{|c|}{ Alcohol-related picture } & \multicolumn{4}{|c|}{ Neutral picture } \\
\hline & & \multicolumn{2}{|l|}{ Go trials } & \multicolumn{2}{|c|}{ No-go trials } & \multicolumn{2}{|l|}{ Go trials } & \multicolumn{2}{|c|}{ No-go trials } \\
\hline & & Lat & Amp & Lat & Amp & Lat & Amp & Lat & Amp \\
\hline \multirow[t]{7}{*}{$\mathrm{HD} / \mathrm{HAA}$} & F3 & 436(98) & $6.9(4.2)$ & $528(135)$ & $10.2(7.7)$ & $484(124)$ & $6.5(7.2)$ & $587(32)$ & $9.0(4.0)$ \\
\hline & $\mathrm{Fz}$ & $426(97)$ & $5.7(3.5)$ & $527(144)$ & $8.4(8.7)$ & 481(119) & $5.2(7.0)$ & $591(44)$ & $9.8(4.1)$ \\
\hline & $\mathrm{F} 4$ & 437(98) & $7.0(3.3)$ & $524(148)$ & $8.6(7.5)$ & $502(120)$ & $7.9(5.7)$ & $589(44)$ & $9.5(3.2)$ \\
\hline & $\mathrm{C} 3$ & 436(99) & $9.6(4.2)$ & $537(138)$ & $12.4(6.8)$ & $501(106)$ & $9.9(6.8)$ & $587(35)$ & $12.8(4.6)$ \\
\hline & $\mathrm{Cz}$ & $439(105)$ & $11.5(5.0)$ & $584(89)$ & $13.7(8.4)$ & $543(59)$ & $10.9(7.4)$ & $589(41)$ & $15.7(4.8)$ \\
\hline & $\mathrm{C} 4$ & $477(67)$ & $10.5(4.5)$ & $589(96)$ & $12.3(7.4)$ & $550(51)$ & $10.7(5.6)$ & $592(44)$ & $13.4(4.7)$ \\
\hline & $\mathrm{Pz}$ & $439(84)$ & $12.5(5.6)$ & $548(74)$ & $15.1(6.3)$ & $522(49)$ & $11.8(4.9)$ & $585(31)$ & $14.9(7.4)$ \\
\hline \multirow[t]{7}{*}{ HD/LAA } & F3 & 431(139) & $6.5(2.3)$ & $535(22)$ & $15.6(4.3)$ & $484(150)$ & $8.4(4.7)$ & $544(38)$ & $12.3(4.2)$ \\
\hline & $\mathrm{Fz}$ & 432(139) & $6.9(3.3)$ & $526(25)$ & $16.8(4.3)$ & $481(146)$ & $9.3(6.1)$ & $538(38)$ & 13.3(3.9) \\
\hline & $\mathrm{F} 4$ & $431(138)$ & $6.6(3.0)$ & $531(21)$ & $13.6(4.1)$ & $478(118)$ & $9.2(5.8)$ & $536(35)$ & $11.2(2.4)$ \\
\hline & $\mathrm{C} 3$ & $472(93)$ & $11.4(4.1)$ & $554(44)$ & $17.6(6.2)$ & $542(68)$ & $12.3(5.1)$ & $548(36)$ & $15.2(4.2)$ \\
\hline & $\mathrm{Cz}$ & 495(63) & $13.0(6.8)$ & $537(21)$ & $20.8(8.3)$ & $533(63)$ & $16.5(9.4)$ & $545(32)$ & $17.9(5.7)$ \\
\hline & $\mathrm{C} 4$ & $495(60)$ & $11.7(4.9)$ & $551(44)$ & $17.4(6.8)$ & $518(56)$ & $14.8(8.2)$ & $551(38)$ & $15.6(5.1)$ \\
\hline & $\mathrm{Pz}$ & $486(66)$ & $16.0(6.5)$ & $555(41)$ & $18.9(7.8)$ & $528(63)$ & $15.9(7.2)$ & $552(43)$ & $17.5(3.9)$ \\
\hline \multirow[t]{7}{*}{ LD/HAA } & F3 & $418(147)$ & $4.5(5.2)$ & $559(136)$ & $8.9(4.6)$ & $560(150)$ & $2.8(2.9)$ & 591(68) & $8.7(7.6)$ \\
\hline & $\mathrm{Fz}$ & $469(160)$ & $4.0(7.1)$ & $559(136)$ & $9.3(7.7)$ & $552(143)$ & $1.7(4.0)$ & $600(83)$ & $9.5(10.2)$ \\
\hline & $\mathrm{F} 4$ & $459(157)$ & $4.9(6.1)$ & $560(136)$ & $9.8(8.1)$ & $549(141)$ & $3.8(4.7)$ & $604(86)$ & $10.5(8.9)$ \\
\hline & $\mathrm{C} 3$ & $366(88)$ & $7.6(7.3)$ & $570(142)$ & $11.4(4.8)$ & $551(148)$ & $6.1(3.3)$ & $606(81)$ & $11.5(6.7)$ \\
\hline & $\mathrm{Cz}$ & $454(160)$ & $7.8(8.3)$ & $468(143)$ & $12.6(7.8)$ & $545(144)$ & $6.4(4.8)$ & $602(81)$ & $12.4(9.1)$ \\
\hline & $\mathrm{C} 4$ & $459(152)$ & $7.9(7.2)$ & $566(143)$ & $11.3(6.2)$ & $529(139)$ & $7.1(5.1)$ & $596(80)$ & $12.2(6.4)$ \\
\hline & $\mathrm{Pz}$ & $391(89)$ & $12.3(6.9)$ & $568(141)$ & $15.0(6.9)$ & 516(119) & $11.5(5.4)$ & $599(76)$ & $15.8(4.8)$ \\
\hline \multirow[t]{7}{*}{ LD/LAA } & F3 & $478(52)$ & $3.8(4.1)$ & $549(51)$ & $12.8(6.7)$ & $533(126)$ & $6.6(4.2)$ & $617(51)$ & $9.5(6.2)$ \\
\hline & $\mathrm{Fz}$ & $446(118)$ & $2.5(4.7)$ & $552(53)$ & $12.9(6.1)$ & $495(150)$ & $5.0(4.7)$ & $617(49)$ & $9.6(6.1)$ \\
\hline & $\mathrm{F} 4$ & $473(106)$ & $5.6(4.2)$ & $557(63)$ & $13.1(5.4)$ & $487(140)$ & $7.0(5.2)$ & $614(51)$ & $11.1(3.4)$ \\
\hline & C3 & $476(55)$ & $9.2(3.9)$ & $565(55)$ & $15.3(4.7)$ & $559(32)$ & $11.6(4.3)$ & $604(36)$ & $12.9(3.4)$ \\
\hline & $\mathrm{Cz}$ & $483(61)$ & $9.4(6.4)$ & $562(50)$ & $16.3(5.9)$ & $560(31)$ & $11.6(6.3)$ & $603(34)$ & 15.1(4.8) \\
\hline & $\mathrm{C} 4$ & $483(57)$ & $9.5(4.9)$ & $583(46)$ & $14.3(4.8)$ & $553(27)$ & $11.4(4.4)$ & $610(38)$ & $13.4(2.8)$ \\
\hline & $\mathrm{Pz}$ & $441(35)$ & $13.5(8.5)$ & $551(102)$ & $16.4(6.1)$ & $521(56)$ & $13.5(6.5)$ & $577(66)$ & $15.9(4.0)$ \\
\hline
\end{tabular}


Table 4

Correlations for BIS-11 scores, AUDIT scores, obsessed craving and the percentage of false alarm to alcohol-related cues in No-go trials; and the mean latency and amplitude of N200 and P300 for alcohol-related cues in No-go trials.

\begin{tabular}{|c|c|c|c|c|c|}
\hline & \multirow[t]{2}{*}{ FAs } & \multicolumn{2}{|c|}{ N200 No-go } & \multicolumn{2}{|c|}{ P300 No-go } \\
\hline & & Lat & Amp & Lat & Amp \\
\hline FAs for alcohol related-cues & & 0.29 & -0.31 & $0.38^{*}$ & 0.01 \\
\hline AUDIT score & 0.06 & -0.13 & 0.02 & -0.04 & 0.17 \\
\hline Impulsivity & $0.43^{*}$ & 0.24 & -0.25 & -0.12 & -0.07 \\
\hline Obsessed craving & 0.30 & -0.06 & 0.07 & -0.06 & -0.31 \\
\hline
\end{tabular}

*Correlation is significant at the 0.05 level.

towards alcohol-related cues in heavy drinkers could be explained by two factors. First, the level of alcohol avoidance, combined with the pattern of consumption, could play a key role in the occurrence of FA. Indeed, we found that high alcohol avoiders performed more FA than low alcohol avoiders independently of stimulus category, and that heavy drinkers who experienced high alcohol avoidance tended to make more FA for alcohol-related cues than those who experienced low alcohol avoidance. This finding is quite surprising because the self-reported level of alcohol avoidance has been found to correlate negatively with automatic approach tendencies (Barkby et al., 2012). However, recent findings report that alcohol-dependent patients inclined to avoid alcohol show higher relapse rates, suggesting that alcohol avoidance could hide approach drives (Spruyt et al., 2013). The second factor that could explain the absence of inhibition deficit towards alcohol-related cues in heavy drinkers is the low impulsivity level found in our participants. This could act as a protective factor against the difficulty to refrain a response toward alcohol (Papachristou et al., 2012). The positive correlation between the impulsivity level and the percentage of FA to alcohol-related cues found in the present study supports this hypothesis.

The present results showed that all the participants were faster to process alcohol-related cues as reflected by earlier P300 latencies during Go trials and by shorter RT. Previous findings also reported faster responses to alcohol-related cues independently of participants' alcohol consumption profile, which could mean the high salient emotional value of alcohol stimuli experienced by all participants (Kreusch et al., 2013).

When alcohol avoidance was included as a between group factor in our ERP analyses, the results showed a larger N200 amplitude tendency in higher alcohol avoiders than in light alcohol avoiders during No-go trials. This non-significant tendency suggests that higher alcohol avoiders recruited larger cognitive control resources when they had to inhibit their responses in the alcohol modified Go/No-go task. Moreover, the results indicated higher N200 amplitude in No-go trials as compared to Go trials among alcohol avoiders, whereas no such differences were found between No-go and Go trials for lower alcohol avoiders. In previous studies, larger N200 amplitude was reported following incongruent conditions such as the avoidance of positive stimuli (Ernst et al., 2013) or the interference by irrelevant information (Yeung and Cohen, 2006). Moreover, several studies indicated that anxious individuals seem to maintain a higher level of cognitive control to cope and to monitor the outcome of their actions reflecting by larger N200 amplitude (Righi et al., 2009; Sehlmeyer et al., 2010). As participants avoiding alcohol were constrained to process alcohol cues, they were probably in an incongruent condition, felt more anxious, leading to the recruitment of higher cognitive control resources in order to perform the task. Our results showed also that low alcohol avoiders exhibited higher P300 amplitude for alcohol-related cues during No-go trials than during Go trials, but no such difference was found in high alcohol avoiders, suggesting lesser reactivity to alcohol cues in higher alcohol avoiders. Such finding (less P300 amplitude reactivity, following by larger N200 amplitude) has previously been associated with a higher task difficulty (Gajewski \& Falkenstein, 2013; Benikos et al., 2013), suggesting that inhibiting a response towards alcohol-related cue would be more difficult in high alcohol avoiders than in low avoiders. Higher alcohol avoiders could allocate more attentionnal resources to inhibit the response (i.e., higher N200 amplitude), leading to less reactivity to alcohol-cues as compared to low alcohol avoiders (i.e., no higher P300 amplitude for alcohol related cues). Taken together, our behavioral and ERP data suggest that the level of alcohol avoidance influences the processing of alcohol-related stimuli more than the current levels of alcohol consumption in non alcohol dependent participants.

Before concluding, some limitations of the present study should be acknowledged. The main limitation is the small sample size that may prevent the detection of some main effects, interactions, and correlations. Secondly, our sample consists of university students, and therefore our results must be extended to other populations of alcohol abusers. Thirdly, since amplitudes of some ERP components can be affected by the family history of alcoholism or substance abuse (Porjesz et al., 2005; Euser et al., 2012), the absence of control over this factor in the present study constitutes a limitation. Fourthly, as the ERPs are recorded only at the median line ( $\mathrm{Fz}, \mathrm{Cz}, \mathrm{Pz})$ and fronto-central sites ( $\mathrm{F}$, $\mathrm{F} 4, \mathrm{C} 3, \mathrm{C} 4$ ) in present study, further studies must be conducted with more electrode sites to allow a better source localization. Finally, we focused only on the N200 and P300 components. However, recent studies found specific modulations of earlier components like the P100 among binge drinkers. Indeed, Petit et al. (2012b) reported greater P100 amplitudes to alcoholic stimuli in comparison with neutral stimuli in young social drinkers, suggesting enhanced perceptual processing toward alcohol cues. Consequently, this study should be considered as a preliminary one and further research using a larger sample of heterogeneous alcohol consumers should be conducted to assess the ability to inhibit a response towards alcohol-related stimuli.

In conclusion, the present results indicate lower inhibition capacities in heavy drinkers as reflected by the absence of N200 anteriorization. When the inhibition capacities towards alcohol-related cues were investigated, the level of alcohol avoidance impacts the processing of such cues. Indeed, participants who experienced higher avoidance for alcohol made more FA, recruited larger attentionnonal resources (i.e., larger N200) and weaker decisional processes (i.e., less P300 reactivity), especially for alcohol-related cues in the modified alcohol Go/No-go task. In a context involving alcohol-related cues, higher alcohol avoiders could therefore be more disturbed by the presence of alcohol-related cues.

\section{Conflict of interest}

None.

\section{Acknowledgments}

This work was supported by the Belgian National Fund for Scientific Research (FNRS). Fanny Kreusch is a FNRS Research Fellow. We thank Fabian Verkenne for providing his help during data collection.

\section{References}

Adams, S.,Ataya, A.F.,Attwood, A.S., Munafò, M.R., 2013. Effects of alcohol on disinhibition towards alcohol-related cues. Drug Alcohol Depend. 127, 137-142.

Barkby, H.,Dickson, J.M.,Roper, L.,Field, M., 2012. To approach or avoid alcohol? Automatic and self-reported motivational tendencies in alcohol dependence. Alcohol. Clin. Exp. Res. 36, 361-368

Barry, R.J.,Rushby, J.A., 2006. An orienting reflex perspective on anteriorisation of the P3 of the event-related potential. Exp. Brain Res. 173, 539-545.

Bartholow, B.D., Henry, E.A., Lust, S.A., 2007. Effects of alcohol sensitivity on P3 eventrelated potential reactivity to alcohol cues. Psychol. Addict. Behav. 21, 555-563.

Bartholow, B.D., Lust, S.A., Tragesser, S.L., 2010. Specificity of P3 event-related potential reactivity to alcohol cues in individuals low in alcohol sensitivity. Psychol. Addict. Behav. 24, 220-228.

Beck, A.T.,Steer, R.A., Brown, G.K., 1996. BDI II, Inventaire de Dépression de Beck, 2e ed. Edition du Centre de Psychologie Appliquée, Paris. 
Benikos, N.,Johnstone, S.J., Roodenrys, 2013. Varying task difficulty in the Go/Nogo task: the effects of inhibitory control, arousal, and perceived effort on ERP components. Int. J. Psychophysiol. 87, 262-272.

Bijl, S., de Bruin, E.A., Böcker, K.B., Kenemans, J.L., Verbaten, M.N., 2005. Chronic effects of social drinking in a card-sorting task: an event related potential study. Clin. Neurophysiol. 116, 376-385.

Bruin, K.J., Wijers, A.A., 2002. Inhibition, response mode, and stimulus probability: a comparative event-related potential study. Clin. Neurophysiol. 113, 1172-1182.

Cohen, H.L., Porjesz, B., Begleiter, H.,Wang, W., 1997. Neurophysiological correlates of response production and inhibition in alcoholics. Alcohol. Clin. Exp. Res. 2, 1398-1406.

Enriquez-Geppert, S.,Konrad, C.,Pantev, C.,Huster, R.J., 2010. Conflict and inhibition differentially affect the N200/P300 complex in a combined go/nogo and stop-signal task. Neurolmage 51, 877-887.

Ernst, L.H., Ehlis, A., Dresler, T., Tupak, S.V.,Weidner, A., Fallgatter, A.J., 2013. N1 and N2 ERPs reflect the regulation of automatic approach tendencies to positive stimuli. Neurosci. Res. 75, 239-249.

Euser, A.S., Franken, I.S., 2012. Alcohol affects the emotional modulation of cognitive control: an event-related brain potential study. Psychopharmacology 222, 459-476.

Euser, A.S., Arends, L.R., Evans, B.E., Greaves-Lord, K., Huizink, A.C., Franken, I.H., 2012. The P300 event-related brain potential as a neurobiological endophenotype for substance use disorders: a meta-analytic investigation. Neurosci. Biobehav. Rev. 36, 572-603.

Fadardi, J.S., Cox, W.M., 2008. Alcohol attentional bias and motivational structure as independent predictors of social.drinkers' alcohol consumption. Drug Alcohol Depend. 97, 247-256

Falkenstein, M.,Hoormann, J., Hohnsbein, J., 1999. ERP components in Go/Nogo tasks and their relation to inhibition. Acta Psychol. 101, 267-291.

Field, M., Cox, W.M., 2008. A review of its development, causes, and consequences. Drug Alcohol Depend. 97, 1-20.

Field, M., Christiansen, P., Cole, J., Goudie, A., 2007. Delay discounting and the alcohol Stroop in heavy drinking adolescents. Addiction 102, 579-586.

Field, M., Kiernan, A., Eastwood, B., Child, R., 2008. Rapid approach responses to alcohol cues in heavy drinkers. J. Behav. Ther. Exp. Psychiatry 39, 209-218.

Field, M., Wiers, R.W., Christiansen, P., Fillmore, M.T., Verster, J.C., 2010. Acute alcohol effects on inhibitory control and implicit cognition: implications for loss of control over drinking. Alcohol. Clin. Exp. Res. 34, 1346-1352.

Fillmore, M.T., 2003. Drug abuse as a problem of impaired control: current approaches and findings. Behav. Cogn. Neurosci. Rev. 2, 179-197.

Fleming, M.F.,Barry, K.L.,MacDonald, R., 1991. The alcohol use disorders identification test (AUDIT) in a college sample. Int. J. Addict. 26, 1173-1185.

Gajewski, P.D.,Falkenstein, M., 2013. Effects of task complexity on ERP components in Go/ Nogo tasks. Int. J. Psychophysiol. 87, 273-278.

Hansenne, M., Olin, C., Pinto, E., Pitchot, W., Ansseau, M., 2003. Event-related potentials to emotional and neutral stimuli in alcoholism. Neuropsychobiology 48, 77-81.

Herrmann, M.J., Weijers, H.G., Wiesbeck, G.A., Aranda, D., Böning, J., Fallgatter, A.J., 2000. Event-related potentials and cue-reactivity in alcoholism. Alcohol. Clin. Exp. Res. 24, 1724-1729.

Herrmann, M.J., Weijers, H.G.,Wiesbeck, G.A., Böning, J., Fallgatter, A.J., 2001. Alcohol cuereactivity in heavy and light social drinkers as revealed by event-related potentials. Alcohol Alcohol. 36, 588-593.

Huster, R.J.,Enriquez-Geppert, S., Lavallee, C.F., Falkenstein, M., Herrmann, C.S., 2013. Electroencephalography of response inhibition tasks: functional networks and cognitive contributions. Int. J. Psychophysiol. 87, 217-233.

Kaiser, S.,Unger, J., Kiefer, M., Markela, J., Mundt, C., et al., 2003. Executive control deficit in depression: event-related potentials in a Go/Nogo task. Psychiatry Res. 122, 169-184.

Kamarajan, C., Porjesz, B., Jones, K.A., Choi, K., Chorlian, D.B., Padmanabhapillai, A., et al., 2005. Alcoholism is a disinhibitory disorder: neurophysiological evidence from a Go/No-go task. Biol. Psychol. 69, 353-373.

Kreusch, F., Vilenne, V., Quertemont, E., 2013. Response inhibition toward alcohol-related cues using an alcohol go/no-go task in problem and non-problem drinkers. Addict. Behav. 38, 2520-2528.

Lavric, A., Pizzagalli, D.A., Forstmeier, S., 2004. When 'go' and 'nogo' are equally frequent: ERP components and cortical tomography. Eur. J. Neurosci. 20, 2483-2488.

López-Caneda, E., Cadaveira, F., Crego, A., Gómez-Suárez, A., Corral, M., Parada, M., Caamaño-Isorna, F., Rodríguez Holguín, S., 2012. Hyperactivation of right inferior frontal cortex in young binge drinkers during response inhibition: a follow-up study. Addiction 107, 1796-1808.

López-Caneda, E., Cadaveira, F.,Crego, A.,Doallo, S.,Corral, M.,Gómez-Suárez, A., Rodríguez Holguín, S., 2013. Effects of a persistent binge drinking pattern of alcohol consumption in young people: a follow-up study using event-related potentials. Alcohol Alcohol. $48,464-471$.

López-Caneda, E.,Rodríguez Holguín, S.,Corral, M.,Doallo, S.,Cadaveira, F., 2014. Evolution of the binge drinking pattern in college students: neurophysiological correlates. Alcohol http://dx.doi.org/10.1016/j.alcohol.2014.01.009.

Maurage, P., Pesenti, M., Philippot, P., Joassin, F., Campanella, S., 2009. Latent deleterious effects of binge drinking over a short period of time revealed only by electrophysiological measures. J. Psychiatry Neurosci. 34, 111-118.

Maurage, P., Joassin, F., Speth, A., Modave, J., Philippot, P., Campanella, S., 2012. Cerebral effects of binge drinking: respective influences of global alcohol intake and consumption pattern. Clin. Neurophysiol. 123, 892-901.

McEvoy, P.M., Stritzke, W.G., French, D.J., Lang, A.R., Ketterman, R., 2004. Comparison of three models of alcohol craving in young adults: a cross-validation. Addiction 99, 482-497.

Montgomery, C.,Fisk, J.E.,Murphy, P.N.,Ryland, I.,Hilton, J., 2013. The effects of heavy social drinking on executive function: a systematic review and meta-analytic study of existing literature and new empirical findings. Hum. Psychopharmacol: Clin. Exp. 27, 187-199.
Namkoong, K.,Lee, E.,Lee, C.H. Lee, B.O.,An, S.K. 2004. Increased P3 amplitudes induced by alcohol-related pictures in patients with alcohol dependence. Alcohol. Clin. Exp. Res. 28, 1317-1323.

Nederkoorn, C., Baltus, M., Guerrieri, R.,Wiers, R.W., 2009. Heavy drinking is associated with deficient response inhibition in women but not in men. Pharmacol. Biochem. Behav. 93, 331-336.

Nichols, J.M., Martin, F., 1996. The effect of heavy social drinking on recall and eventrelated potentials. J. Stud. Alcohol Drugs 57, 125-135.

Nieuwenhuis, S., Yeung, N.,van den Wildenberg, W., Ridderinkhof, R., 2003. Electrophysiological correlates of anterior cingulated function in a go/nogo task: effects of response conflict and trial type frequency. Cogn. Affect. Behav. Neurosci. 3, 17-26.

Nigg, J.T., Wong, M.M., Martel, M.M., Jester, J.M., Puttler, L.I., Glass, J.M., et al., 2006. Poor response inhibition as a predictor of problem drinking and illicit drug use in adolescents at risk for alcoholism and other substance use disorders. J. Am. Acad. Child Adolesc. Psychiatry 45, 468-475.

Noël, X., Van der Linden, M., d'Acremont, M., Bechara, A., Dan, B., Hanak, C., et al., 2007. Alcohol cues increase cognitive impulsivity in individuals with alcoholism. Psychopharmacology 192, 291-298.

Noël, X.,Bechara, A.,Brevers, D.,Verbanck, P.,Campanella, S., 2010. Alcoholism and the loss of willpower: a neurocognitive perspective. J. Psychophysiol. 24, 240-248.

Oddy, B.W., Barry, R.J., 2009. The relationship of N2 and P3 to inhibitory processing of social drinkers in a Go/NoGo task. Int. J. Psychophysiol. 72, 323-330.

Pandey, A.K., Kamarajan, C., Tang, Y., Chorlian, D.B., Roopesh, B.N., Manz, N., Stimus, A., Rangaswamy, M.,Porjesz, B., 2012. Neurocognitive deficits in male alcoholics: an ERP/sLORETA analysis of the N2 component in an equal probability Go/NoGo task Biol. Psychol. 89, 170-182.

Papachristou, H., Nederkoorn, C., Havermans, R.,van der Horst, M., Jansen, A., 2012. Can't stop the craving: the effect of impulsivity on cue-elicited craving for alcohol in heavy and light social drinkers. Psychopharmacology 219, 511-518.

Parsons, O.A., 1998. Neurocognitive deficits in alcoholics and social drinkers : a continuum? Alcohol. Clin. Exp. Res. 22, 954-961.

Petit, G.,Kornreich, C.,Noël, X.,Verbanck, P.,Campanella, S., 2012a. Alcohol-related context modulates performance of social drinkers in a visual Go/No-go task: a preliminary assessment of event-related potentials. PLoS ONE 7, e37466.

Petit, G., Kornreich, C., Maurage, P., Noël, X., Letesson, C., Verbanck, P., et al., 2012b. Early attentional modulation by alcohol-related cues in young binge drinkers: an eventrelated potentials study. Clin. Neurophysiol. 123, 925-936.

Porjesz, B., Rangaswamy, M., Kamarajan, C., Jones, K.A., Padmanabhapillai, A., Begleiter, H., 2005. The utility of neurophysiological markers in the study of alcoholism. Clin. Neurophysiol. 116, 993-1018.

Righi, S., Mecacci, L., Pia Viggiano, M., 2009. Anxiety, cognitive self-evaluation and performance: ERP correlates. J. Anxiety Disord. 23, 1132-1138.

Robinson, T.E.,Berridge, K.C. 1993. The neural basis of drug craving: an incentive sensitization theory of addiction. Brain Res. Rev. 18, 247-291.

Saunders, J.B., Aasland, O.G., Babor, T.F., de la Fuente, J.R., Grant, M., 1993. Development of the Alcohol Use Disorders Identification Test (AUDIT): WHO collaborative project on early detection of persons with harmful alcohol consumption-II. Addiction 88 791-804.

Sehlmeyer, C., Konrad, C., Zwitserlood, P., Arolt, V., Falkenstein, M., Beste, C., 2010. ERP indices for response inhibition are related to anxiety-related personality traits. Neuropsychologia 48, 2488-2495.

Smith, J.L.,Johnstone, S.J.,Barry, R.J., 2007. Response priming in the Go/NoGo task: the N2 reflects neither inhibition nor conflict. Clin. Neurophysiol. 118, 343-355.

Smith, J.L., Johnstone, S.J., Barry, R.J., 2008. Movement-related potentials in the Go/NoGo task: the P3 reflects both cognitive and motor inhibition. Clin. Neurophysiol. 119, 704-714.

Smith, J.L., Smith, E.A., Provost, A.L., Heathcote, A., 2010. Sequence effects support the conflict theory of N2 and P3 in the Go/NoGo task. Int. J. Psychophysiol. 75, 217-226.

Sobell, L.C., Sobell, M.B., 1990. Self-report issues in alcohol abuse: state of the art and future directions. J. Psychopathol. Behav. Assess. 12, 77-90.

Spielberger, C.D., 1983. Manual for the State-Trait Anxiety Inventory STAI (Form Y). Mind Garden, Palo Alto, CA.

Spruyt, A., De Houwer, J., Tibboel, H., Verschuere, B., Crombez, G., Verbanck, P., Hanak, C., Brevers, D., Noël, X., 2013. On the predictive validity of automatically activated approach/avoidance tendencies in abstaining alcohol-dependent patients. Drug Alcohol Depend. 127, 81-86.

Stacy, A., Wiers, R.W., 2010. Implicit cognition and addiction : a tool for explaining paradoxical behavior. Annu. Rev. Clin. Psychol. 6, 551-575.

Townshend, J.M.,Duka, T., 2001. Attentional bias associated with alcohol cues: differences between heavy and occasional social drinkers. Psychopharmacology 157, 67-74.

Watson, T.D., Sweeney, J.F., Louis, H., 2014. Neurocognitive, psychological and behaviora correlates of binge drinking and use of alcohol with caffeinated beverages in college-aged adults. Am. J. Drug Alcohol Abuse 40, 58-66.

Wiers, R.W., Hoogeveen, K., Sergeant, J.A., Gunning, B.W., 1997. Hight and low-dose alcohol-related expectancies and the differential associations with drinking in male and female adolescents and young adults. Addiction 92, 871-888.

Wiers, R.W.,Bartholow, B.D.,van den Wildenberg, E.Thush, C., Engels, R.C.,Sher, K.J., et al, 2007. Automatic and controlled processes and the development of addictive behaviors in adolescents : a review and a model. Pharmacol. Biochem. Behav. 86, 263-283.

Yeung, N., Cohen, J.D., 2006. The impact of cognitive deficits on conflict monitoring: predictable dissociations between the error-related negativity and N2. Psychol. Sci. 17, 164-171. 